\title{
Evaluation of certain bioactive ingredients of $\gamma$-irradiated tomato pomace and its effects on biochemical attributes of Wister male rats
}

\author{
Diaa El-Din H. Farag ${ }^{1}$, Dina M. Seoudi², Manal A. Emam², Nahla S. Hassan² and Ashraf M. \\ Mounir $^{1}$ \\ ${ }^{1}$ National Center for Radiation Research and Technology, Atomic Energy Authority, Egypt \\ ${ }^{2}$ Department of Biochemistry, Faculty of Science, Ain Shams University, Abbassia 11566, Cairo, Egypt.
}

\section{ARTICLE INFO}

Article history:

Received 31 January 2016

Accepted 21 February 2016

Keywords:

Tomato pomace;

$\gamma$-irradiation;

antioxidants.

\begin{abstract}
A B S T R A C T
Food irradiation is a method of preservation; it is used to extend the shelf life of food products fresh and/or dried, destroy the contaminating harmful pathogens and modify the activity of bioactive compounds present in food materials. The present study was conducted to test the possible biochemical impacts of $\gamma$ irradiation on tomato pomace, in addition to elucidate the physiological and biochemical effects of feeding Wister rats diets supplemented with irradiated tomato pomace at dose levels of 10,20 and $30 \mathrm{kGy}$. The chemical composition of processed tomato pomace at the above mentioned doses have no significant differences compared to the non-irradiated one. The results indicated that irradiation treatment up to $30 \mathrm{kGy}$ caused a significant reduction in scavenging ability of free radicals, reduction in tannins content, and increase in polyphenols as a function of radiation dose. Both amino acids pattern and fatty acids profile were significantly affected by irradiation processing without a specific trend. Feeding Wister rats with high fat diets supplemented with non-irradiated or irradiated up to $30 \mathrm{kGy}$-tomato pomace for 8 weeks, showed better results when compared with those rats fed on reference diet. Body weight gain and internal organ weight alongside some biochemical parameters such as serum total cholesterol, triglycerides, lipoproteins, liver enzymes and plasma glucose revealed better results upon the same mentioned comparisons.
\end{abstract}

\section{Introduction}

During endogenous metabolic reactions, aerobic cells produce reactive oxygen species (ROS) such as superoxide anion $\left(\mathrm{O}_{2}^{-}\right)$, hydrogen peroxide $\left(\mathrm{H}_{2} \mathrm{O}_{2}\right)$, hydroxyl radical $\left(\mathrm{OH}^{-}\right)$, and organic peroxides as normal products of the biological reduction of molecular oxygen. They have several important physiological functions, but their accumulation beyond the needs of the cell can potentially damage lipids, proteins, and nucleic acids ${ }^{[1,2]}$.

Presence of excess ROS causes oxidative stress, the continued oxidative stress can lead to chronic inflammation, which in turn could mediate most chronic diseases including cancer, diabetes, cardiovascular, neurological, aging and pulmonary diseases ${ }^{[3,4]}$. In such conditions, external supply of antioxidants is essential to countervail the deleterious consequences of oxidative stress ${ }^{[5]}$.

\footnotetext{
* Corresponding author.

E-mail address: diaaf@hotmail.com
}

The processing of plant foods results in the production of by-products that are rich sources of bioactive compounds, including phenolic compounds ${ }^{[6,7,8]}$. Phenolic compounds with antioxidant activity have been identified in several agricultural by-products, such as rice hulls ${ }^{[9]}$, buckwheat hulls ${ }^{[10]}$ and almond hulls ${ }^{[11]}, 26$ different fruit and vegetable wastes ${ }^{[12]}$.

Tomato (Lycopersicum esculentum) is one of the most consumed vegetables in the world, either as a raw fruit or as a processed product. Tomatoes are used in the foodstuff industry as raw material for the production of several products such as juices, sauces, purees, pastes, and canned tomatoes ${ }^{[13]}$.

The peel of tomato is available in large quantities as a by-product, it was estimated that about $1.25 \%$ solid wastes produced from processing raw tomato, more than 16 million metric tons of tomatoes were estimated to have been processed in the EU in 2005, leading to more than 200,000 tons of solid tomato residue ${ }^{[13]}$. According to Horticulture Research Institute-Giza-Egypt in 2014 about 7567 acres were planted with tomato in Egypt. 
Lycopene present in raw tomato and its by-products used as a food/dietary supplement in products where the presence of lycopene provides a specific value (e.g., antioxidant or other claimed health benefits) ${ }^{[14]}$.

Conventional methods of microbial decontamination are fumigation with either gaseous ethylene oxide or methyl bromide, which are carcinogenic and hence are now prohibited according to Montreal protocol or being increasingly restricted in most advanced countries for health, environmental or occupational safety reasons. The most advanced method used currently to eliminate pathogen from food, feed and agro-industrial wastes is gamma irradiation process. It is well known as a decontamination method for many food stuffs and plant materials, being an environment friendly and effective technology to resolve technical problems in trade and commercialization $^{[15]}$.

\section{Materials and Methods \\ Materials}

Tomato pomace from Heinz factory in 6-October city, Egypt.

\section{Preparation of Samples}

Fresh samples were dried in shade under continuous air using electrical fan, at room temperature $\left(25^{\circ} \mathrm{C}\right)$ and grounded to the finest particles.

\section{Radiation Processing}

Dry grounded samples were packed in well-sealed polyethylene sleeves (1000 gauge, $0.25 \mathrm{~mm}$ thickness). They were subjected at room temperature to gamma irradiation at dose levels of $0,10,20$ and $30 \mathrm{kGy}$, at dose level $10 \mathrm{kGy} / 3$ hours as monitored by FWT-60$00^{\mathrm{TM}}$ radio chromic film. The radio-chromic dosimeter was purchased from Far West Technology, Inc., Goleta, California, USA, (ASTM, 2002 [ISO/ASTM 51275:2002(E)]). The irradiation facility used was Egypt's Mega Gamma-1 Type J-65000 located at the National Center for Radiation Research and Technology (NCRRT), Nasr City, Cairo, Egypt. The non-irradiated and irradiated samples were stored at $4^{\circ} \mathrm{C}$ until used.

\section{Animals}

A total number of 40 Wister male rats with initial weight of 100-120 g were obtained from animal house of National Center for Radiation Research and Technology, Cairo, Egypt; animals were examined by veterinary. The animals were healthy and clinically free of internal and external parasites. Animals were kept in the animal house at an ambient temperature $22{ }^{\circ} \mathrm{C} \pm 3$ and $50 \pm 5 \%$ relative humidity with a $12 \mathrm{~h}$ each of dark and light cycle and water ad libitum, all groups were adapted for one week under standard ration meets their nutritional requirements before the experiment.

\section{Feeding experiment}

Rats were individually weighted and assigned into 6 groups each group consists of 5-7 rats, each group were assigned for specific diet, D1: Reference diet (Table 1), D2: Reference diet plus $20 \%$ fat, D3: High fat diet incorporated with non-irradiated tomato pomace, D4: High fat diet incorporated with $10 \mathrm{kGy}$ irradiated tomato pomace, D5: High fat diet incorporated with $20 \mathrm{kGy}$ irradiated tomato pomace, D6: High fat diet incorporated with $30 \mathrm{kGy}$ irradiated tomato pomace, rats kept on these diets for 8 weeks, At the end of the experimental period ( 8 weeks) blood was obtained by cardiac puncture using a 20 gauge needle according to the method reported by Dacie and Lewis ${ }^{[16]}$, and blood samples from each rat were collected in two sterile test tubes, one contain sodium fluoride to estimate plasma glucose, the other containing blood samples was centrifuged at $5000 \mathrm{rpm}$ for 10 minutes, then serum was separated and collected.

\section{Methods}

\section{Chemical composition}

Moisture content, crude fat, crude protein, ash content was determined according to the standard methods described in the A.O.A.C. [17], crude fiber was determined according to standard method described in the A.O.A.C ${ }^{[18]}$, and nitrogen free extract (NFE) was calculated according to the equation

NFE $(\%)=100-(\%$ Protein $+\%$ Fat $+\%$ Crude Fiber + $\%$ Ash $+\%$ Moisture).

Lipid extraction and determination of fatty acids methyl esters by GLC

The lipid content of dried ground non-irradiated and irradiated tomato pomace was extracted using diethyl ether. The free fatty acids were methylated by methylating agent according to Hamilton and Hamilton [19]. Fatty acids methyl esters were dissolved in chloroform (HPLC grade) and chromatographic separation was performed using hp 6890 gas chromatograph instrument equipped with a flame ionization detector using innowax-cross linked polyethylene glycol fused silica column (30 m long, 0.32 $\mathrm{mm}$ i.d. $0.5 \mu \mathrm{m}$ film thickness). Oven temperature was programmed from $150^{\circ} \mathrm{C}$ for 1 min then elevated to $235^{\circ} \mathrm{C}$ with a rate of $17^{\circ} \mathrm{C} / \mathrm{min}$ then was raised again to $245^{\circ} \mathrm{C}$ with a rate of $11^{\circ} \mathrm{C} / \mathrm{min}$ and hold at $245^{\circ} \mathrm{C}$ for 5 min. gasses flow rates for $\mathrm{N}_{2}$ as a carrier gas, $\mathrm{H}_{2}$ and air were $1.3,40$ and $400 \mathrm{ml} / \mathrm{min}$, respectively. Flame ionization detector and injection temperatures were $275^{\circ} \mathrm{C}$ and $260^{\circ} \mathrm{C}$, respectively.

\section{Amino acids analysis}

A known amount of the defatted sample (50 mg) was hydrolyzed with HCL $(5 \mathrm{ml}, 6 \mathrm{~N})$ and heated in sealed test tube at $110^{\circ} \mathrm{C}$ for $24 \mathrm{~h}$ according to Suzanna ${ }^{[20]}$. The contents of each tube were filtered, evaporated until dryness and a suitable volume of sodium citrate buffer $(\mathrm{pH} 2.2)$ was added to dissolve the hydrolyzed sample followed by ultra-filtration using a $0.2 \mu \mathrm{m}$ membrane filter [21]. Analyses were performed using high performance amino acids analyzer (Biochrom 20, Auto sampler version; Pharmacia Biotech). Detection was performed at two wave lengths (570 and $440 \mathrm{~nm}$ ). The data of each chromatogram was analyzed by EZ chrome $^{\mathrm{TM}}$ chromatography data system tutorial and user guide-version 6.7 .

\section{Tannins analysis}

It was determined according to, Balbaa ${ }^{[22]}$. 
Free Radical Scavenging ability (DPPH assay)

The antioxidant activity of tomato pomace methanol extract was determined based on the radical scavenging ability in reacting with a stable radical 2, 2-diphenyl-1picrylhydrazyl (DPPH) according to Blois ${ }^{[23]}$.

\section{Total Phenolic compounds}

Extraction and determination of Phenolic compounds was conducted according to the method described by Daniel and George ${ }^{[24]}$.

Biochemical assays

Serum ALT and AST were determined according to Sherwin and Pesce ${ }^{[25]}$. Determination of total serum cholesterol was done according to Richmond ${ }^{[26]}$, Serum HDL-Cholesterol [27], serum triglycerides was determined according to Mgowan et al., ${ }^{[28]}$, serum low density lipoprotein cholesterol (LDL-C) was calculated by the equation: Serum LDL-C $(\mathrm{mg} / \mathrm{dl})=$ total cholesterol- HDL-C - triglycerides/5 ${ }^{[29]}$, serum very low density lipoprotein cholesterol (VLDL-C) was calculated by the equation: Serum VLDL-C $\mathrm{mg} / \mathrm{dl}=$ Triglycerides $/ 5$ [30]. Atherogenic Index (AI) was calculated using the equation, $\mathrm{AI}=\left(\right.$ total cholesterol-HDL-C)/HDL-C ${ }^{[31]}$, Plasma glucose was measured by the method of Caraway and Watts, ${ }^{[32]}$.

\section{Statistical analysis}

The data were subjected to analysis of variance (ANOVA) with one-way classification. Linear regression analysis was utilized to define the relationship between different parameters and irradiation dose (kGy). All analyses were conducted using the general linear model procedure of the Statistical Analysis System Institute, Inc., (SAS, 2003), where appropriate treatment means was separated using the Duncan's Multiple Range Test ${ }^{[33]}$. The $\alpha$-level for significance was $\mathrm{P} \leq 0.05$.

Table 1: Composition of reference diet (maize/soybean) diet

\begin{tabular}{|c|c|}
\hline Ingredients & $\mathrm{g} \mathrm{kg}^{-1}$ \\
\hline Maize & 600 \\
\hline Soybean meal & 200 \\
\hline Wheat bran, fine & 20 \\
\hline Layer Concentrate, (40\% Protein) & 116 \\
\hline Sunflower oil & 16 \\
\hline Limestone & 43 \\
\hline Salt, iodized & 2 \\
\hline Mineral premix ${ }^{1}$ & 1 \\
\hline Vitamin premix ${ }^{2}$ & 2 \\
\hline Total & 1000 \\
\hline \multicolumn{2}{|l|}{ Calculated analysis } \\
\hline Metabolizable energy, $\mathrm{MJ} \mathrm{kg}^{-1}$ & 12.15 \\
\hline Crude protein, $\mathrm{g} \mathrm{kg}^{-1}$ & 215 \\
\hline Available phosphate, $\mathrm{g} \mathrm{kg}^{-1}$ & 4.5 \\
\hline Calcium, $\mathrm{g} \mathrm{kg}^{-1}$ & 25.2 \\
\hline Lysine, $\mathrm{g} \mathrm{kg}^{-1}$ & 10.9 \\
\hline Methionine, $\mathrm{g} \mathrm{kg}^{-1}$ & 4.5 \\
\hline Methionine + Cystine, $\mathrm{g} \mathrm{kg}^{-1}$ & 7.7 \\
\hline
\end{tabular}

${ }^{1}$ Supplied per kg of diet: $12 \mathrm{mg}$ of Cu, $98 \mathrm{mg}$ of Fe, $50 \mathrm{mg}$ of $\mathrm{Zn}, 80 \mathrm{mg}$ of Mn, $0.35 \mathrm{mg}$ of I and $0.2 \mathrm{mg}$ of Co.

${ }^{2}$ Supplied per kg of diet: $8000 \mathrm{IU}$ of vitamin A, $200 \mathrm{IU}$ of vitamin $\mathrm{D}_{3}, 40 \mathrm{IU}$ of vitamin E, $4.5 \mathrm{mg}$ of vitamin $\mathrm{B}_{1}, 9 \mathrm{mg}$ of vitamin $\mathrm{B}_{2}, 9 \mathrm{mg}$ of Vitamin $\mathrm{B}_{6}, 0.04 \mathrm{mg}$ of vitamin $\mathrm{B}_{12}, 85 \mathrm{mg}$ of niacin, $27 \mathrm{mg}$ of D-pantothenic acid, $0.4 \mathrm{mg}$ of biotin, and $1.2 \mathrm{mg}$ of folic acid.

\section{Results}

Regression analysis of the chemical composition of raw and irradiated tomato pomace in Table (2) and Figure (1) showed that crude protein, fat and moisture of irradiated samples at all dose levels were not significantly different from those raw samples, ash content showed ununify changes but still acceptable, however, crude fiber exhibited significant decreased with the applied radiation doses $(10,20$ and $30 \mathrm{kGy})$ by $6.48 \mathrm{~g} . \mathrm{kg}^{-1}$ for each kGy of $\gamma$-rays, following the regression equation representing this effect: crude fiber $\left(\mathrm{g} \cdot \mathrm{kg}^{-1}\right)=515 \cdot 2-6.48 \mathrm{X},(\mathrm{X}=$ radiation dose in $\mathrm{kGy}, \mathrm{R} 2=$ 0.92 and p-value $<0.05$ ), subsequently and the content of nitrogen free extract was increased gradually for each kGy by 5.4 g.kg ${ }^{-1},(\mathrm{R} 2=0.955$ and p-value $<0.05)$.

Table (3) represents the levels of phenolic compounds and tannins alongside DPPH scavenging activity. Results showed that there was a significant change in three tested parameters as a result of radiation processing (Figure 2). Total phenolic compounds were increased as a result of radiation processing in all samples, when compared with non-irradiated tomato pomace. Tannin content of tomato 
pomace processed by 20 and $30 \mathrm{kGy}$ was decreased; as compared with those for raw samples $\left(0.03 \mathrm{mg} . \mathrm{g}^{-1}\right)$. On the other hand, there is no change for tannin content of samples processed by $10 \mathrm{kGy}$ of $\gamma$-rays. All $\gamma$-irradiated samples showed a decrease in DPPH scavenging activity when compared with those recorded for raw samples $(21.6 \%)$.

Table 2: Chemical composition of raw and gamma irradiated tomato pomace

\begin{tabular}{|c|c|c|c|c|c|c|}
\hline \multirow{2}{*}{ Component $\left(\mathrm{g} \mathrm{kg}^{-1}\right)$} & \multicolumn{4}{|c|}{ Dose (kGy) } & \multirow{2}{*}{ Pooled SE } & \multirow{2}{*}{ P-Value } \\
\hline & $\mathbf{0}$ & 10 & 20 & 30 & & \\
\hline Proteins & $213.3^{\mathrm{b}} \pm 0.33$ & $228.3^{\mathrm{ab}} \pm 0.33$ & $216.8^{\mathrm{ab}} \pm 11.40$ & $238.3^{\mathrm{a}} \pm 0.25$ & 6.1008 & N.S. \\
\hline Fiber & $532.0^{\mathrm{a}} \pm 2.31$ & $416.0^{\mathrm{b}} \pm 1.15$ & $404.0^{c} \pm 0.12$ & $320.0^{\mathrm{d}} \pm 1.73$ & 1.5556 & $<0.001$ \\
\hline Ash & $55.5^{\mathrm{b}} \pm 0.12$ & $54.5^{\mathrm{c}} \pm 0.00$ & $57.2^{\mathrm{a}} \pm 0.12$ & $53.7^{\mathrm{d}} \pm 0.12$ & 0.1014 & $<0.001$ \\
\hline Fat & $152.1^{\mathrm{a}} \pm 1.46$ & $155.0^{\mathrm{a}} \pm 2.47$ & $154.8^{\mathrm{a}} \pm 3.03$ & $158.1^{\mathrm{a}} \pm 1.79$ & 2.2707 & N.S. \\
\hline Moisture & $13.6^{\mathrm{a}} \pm 0.73$ & $14.6^{\mathrm{a}} \pm 0.21$ & $12.9^{\mathrm{a}} \pm 0.51$ & $13.3^{\mathrm{a}} \pm 0.91$ & 0.6438 & N.S. \\
\hline Nitrogen Free Extract & $47.1^{\mathrm{d}} \pm 1.45$ & $136.2^{c} \pm 2.40$ & $167.1^{\mathrm{b}} \pm 0.84$ & $217.1^{\mathrm{a}} \pm 0.45$ & 4.7223 & $<0.001$ \\
\hline
\end{tabular}

Values are means \pm SE.

Means in the same Raw with the same letter are not significantly different.

Table 3: Antioxidant content and activity of raw and gamma irradiated tomato pomace

\begin{tabular}{|c|c|c|c|c|c|c|}
\hline \multirow{2}{*}{ Antioxidant Parameter } & \multicolumn{4}{|c|}{ Dose (kGy) } & \multirow{2}{*}{ Pooled SE } & \multirow{2}{*}{ P-Value } \\
\hline & $\mathbf{0}$ & 10 & 20 & 30 & & \\
\hline $\begin{array}{l}\left.\text { Total Phenolic Compounds (mg. } \mathrm{g}^{-1}\right) \\
\text { Change }(\%)\end{array}$ & $0.08^{\mathrm{d}} \pm 0.001$ & $\begin{array}{l}0.33^{\mathrm{a}} \pm 0.001 \\
(+312.50)\end{array}$ & $\begin{array}{l}0.2^{\mathrm{b}} \pm 0.002 \\
(+150.00)\end{array}$ & $\begin{array}{l}0.18^{\mathrm{c}} \pm 0.001 \\
(+125.00)\end{array}$ & 0.0003 & $<0.001$ \\
\hline $\begin{array}{l}\text { Tannins }\left(\mathrm{mg} \mathrm{g}^{-1}\right) \\
\text { Change }(\%)\end{array}$ & $0.03^{\mathrm{a}} \pm 0.001$ & $\begin{array}{l}0.03^{\mathrm{a}} \pm 0.002 \\
(0.00)\end{array}$ & $\begin{array}{l}0.01^{\mathrm{b}} \pm 0.001 \\
(-66.67)\end{array}$ & $\begin{array}{l}0.013^{\mathrm{b}} \pm 0.002 \\
(-56.67)\end{array}$ & 0.0005 & $<0.001$ \\
\hline $\begin{array}{l}\text { DPPH }(\%) \\
\text { Change }(\%)\end{array}$ & $21.6^{\mathrm{a}} \pm 0.28$ & $\begin{array}{l}2.52^{\mathrm{d}} \pm 0.10 \\
(-88.33)\end{array}$ & $\begin{array}{l}8.29^{\mathrm{b}} \pm 0.09 \\
(-61.62)\end{array}$ & $\begin{array}{l}5.87^{\mathrm{c}} \pm 0.08 \\
(-72.82)\end{array}$ & 1.6195 & $<0.001$ \\
\hline
\end{tabular}

Values are means \pm SE.

Means in the same Raw with the same letter are not significantly different.

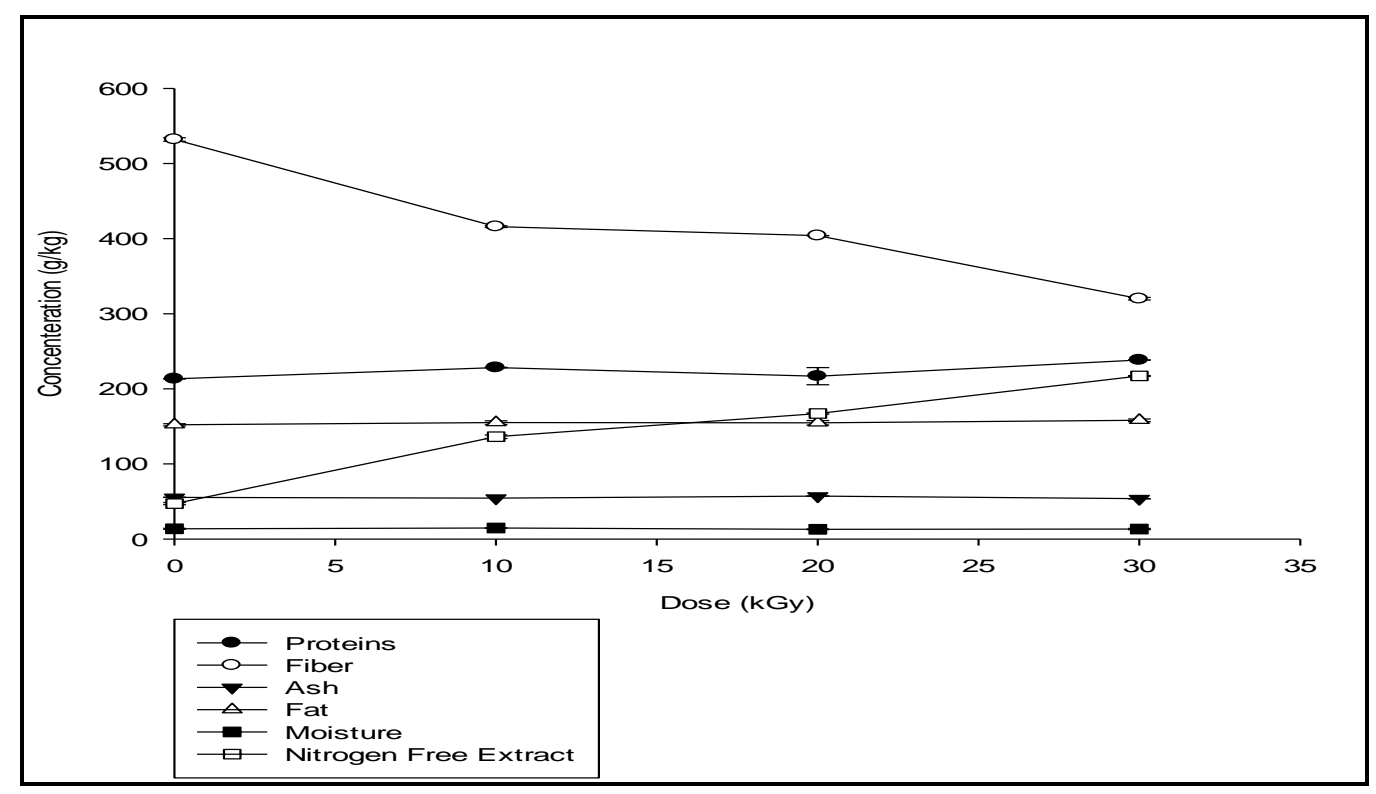

Figure (1): Chemical composition of raw and gamma irradiated tomato pomace 


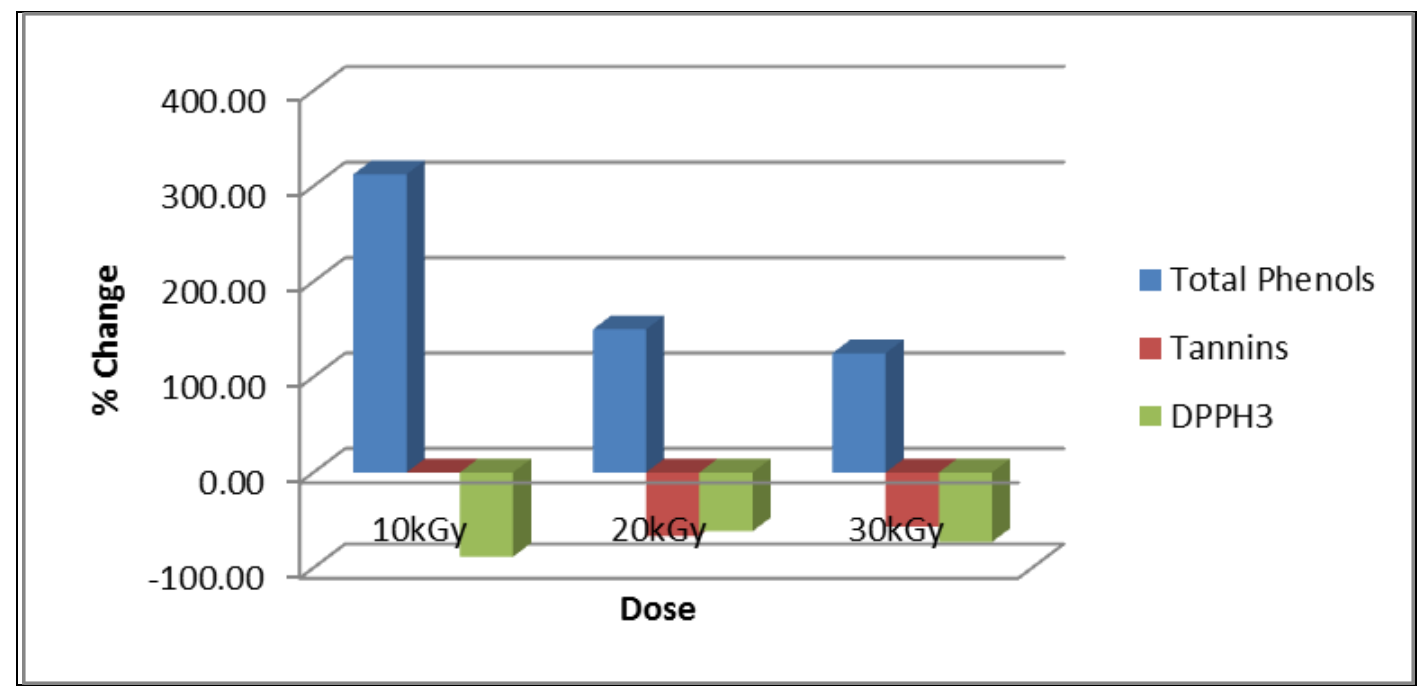

Figure (2): Percent change of antioxidant parameters of $\gamma$-irradiated tomato pomace compared to non-irradiated one

As shown in Table (4), the amino acids content in raw and $\gamma$-irradiated tomato pomace at dose level $10 \mathrm{kGy}$ showed that aspartic, threonine, serine, glutamic, proline, methionine, total amino acids (TAA) and total non-essential amino acids (TNEAA) were decreased while; alanine, cystine, valine, isoleucine, leucine, tyrosine, phenylalanine, histidine, lysine, arginine, total essential amino acids (TEAA), essential amino acids index (EAAI) and biological value (BV) were increased, as compared with the corresponding value of raw sample.
Most tested amino acids and calculated parameters of tomato pomace processed at dose level $20 \mathrm{kGy}$ of $\gamma$-rays were increased, with an exception for serine, glycine, cystine and TNEAA were decreased, while; aspartic, proline, leucine and arginine showed no change from raw samples. Most tested amino acids of tomato pomace processed at dose level $30 \mathrm{kGy}$ of $\gamma$-rays were increased except for aspartic, serine, glutamic, glycine and arginine were unchanged.

Table 4: Amino acids composition of raw and gamma irradiated tomato pomace

\begin{tabular}{|c|c|c|c|c|c|c|c|}
\hline \multirow{2}{*}{ Amino acid } & \multirow{2}{*}{$\begin{array}{l}\text { Control } \\
\mathrm{g} / 100 \mathrm{~g}\end{array}$} & \multicolumn{2}{|c|}{10 KGy } & \multicolumn{2}{|c|}{20 KGy } & \multicolumn{2}{|c|}{30 KGy } \\
\hline & & $\mathrm{g} / 100 \mathrm{~g}$ & \% Change & $\mathrm{g} / \mathbf{1 0 0 g}$ & $\%$ Change & $\mathrm{g} / 100 \mathrm{~g}$ & \% Change \\
\hline Aspartic & 1.3 & 1.2 & -7.69 & 1.3 & 0.00 & 1.3 & 0.00 \\
\hline Threonine & 1.15 & 1.0 & -13.04 & 1.2 & +4.35 & 1.2 & +4.35 \\
\hline serine & 1.05 & 0.8 & -23.81 & 1 & -4.76 & 1.05 & 0.00 \\
\hline Glutamic & 1.4 & 0.00 & -100.00 & 1.5 & +7.14 & 1.4 & 0.00 \\
\hline Proline & 0.65 & 0.1 & -84.62 & 0.65 & 0.00 & 0.75 & +15.38 \\
\hline Glycine & 0.85 & 0.85 & 0.00 & 0.55 & -35.29 & 0.85 & 0.00 \\
\hline Alanine & 0.7 & 0.75 & +7.14 & 0.75 & +7.14 & 0.75 & +7.14 \\
\hline Cystine & 0.8 & 0.95 & +18.75 & 0.75 & -6.25 & 0.95 & +18.75 \\
\hline Valine & 1.1 & 1.15 & +4.55 & 1.15 & +4.55 & 1.2 & +9.09 \\
\hline Methionine & 0.5 & 0.3 & -40.00 & 0.55 & +10.00 & 0.7 & +40.00 \\
\hline Isoleucine & 1.3 & 1.45 & +11.54 & 1.4 & +7.69 & 1.65 & +26.92 \\
\hline Leucine & 3.7 & 3.95 & +6.76 & 3.7 & 0.00 & 3.9 & +5.41 \\
\hline Tyrosine & 1.35 & 1.5 & +11.11 & 1.45 & +7.41 & 1.65 & +22.22 \\
\hline Phenylalanine & 1.4 & 1.5 & +7.14 & 1.45 & +3.57 & 1.6 & +14.29 \\
\hline Histidine & 1.2 & 1.3 & +8.33 & 1.25 & +4.17 & 1.55 & +29.17 \\
\hline Lysine & 1.45 & 1.5 & +3.45 & 1.5 & +3.45 & 1.6 & +10.34 \\
\hline Arginine & 1.8 & 1.85 & +2.78 & 1.8 & 0.00 & 1.8 & 0.00 \\
\hline Total Amino Acids & 21.7 & 20.1 & -7.37 & 21.95 & +1.15 & 24 & +10.60 \\
\hline TEAA & 13.6 & 14.0 & +2.94 & 14 & +2.94 & 15.2 & +11.76 \\
\hline TNEAA & 8.1 & 6.15 & -24.07 & 7.95 & -1.85 & 8.7 & +7.41 \\
\hline EAAI & 24.79 & 25.49 & +2.82 & 25.55 & +3.07 & 28.16 & +13.59 \\
\hline BV & 19.85 & 20.65 & +4.03 & 20.72 & +4.38 & 23.7 & +19.40 \\
\hline
\end{tabular}

TEAA=Total Essential Amino Acid, TNEAA=Total Non-Essential Amino Acid, EAAI=Essential Amino Acid Index, $\mathrm{BV}=$ Biological Value. 
Results of fatty acids content in raw and processed tomato pomace in Table (5) showed the presence of palmetic, stearic, oleic and linoleic acids. Both palmetic and stearic acids are decreased in samples processed by $\gamma$-rays at dose levels 10 and $20 \mathrm{kGy}$, and increased in samples exposed to $30 \mathrm{kGy}$ of $\gamma$ - rays. Regarding to oleic acid irradiation treatment at dose level $10 \mathrm{kGy}$ reduced its content. However on increasing radiation dose an increase in its level occurred for samples processed by 20 and $30 \mathrm{kGy}$, corresponding to its content in non-irradiated sample. Linoleic acid content showed an increase in samples processed by $10 \mathrm{kGy}$ and decrease in both samples processed by 20 and $30 \mathrm{kGy}$.

Results of weight parameters of rats fed on reference, high fat reference, high fat supplemented with irradiated or non- irradiated tomato pomace diets (Table 6), showed that initial weight (IW) of all groups was not significantly different, rats from group D2 showed higher final weight $(\mathrm{FW})$, total weight gain (TWG) and daily weight gain (DWG). For FW of rats in group D1, it showed the smallest weight and FW of rats in group D2 up to group D6; showed higher values and it was not significantly different. Results of TWG, rats in group D1 had the smallest weight followed by group D6, there was no significant difference between rats in groups D2, D3, D4 and D5 and there was no significance between rats from groups D3, D5 and D6, the same pattern was found for DWG as TWG.

Results of relative weight of organs of rats fed on reference, high fat reference, high fat supplemented with irradiated or non- irradiated tomato pomace diets (Table 7), showed that there was no significant difference between relative weights of heart, spleen and testis of rats from all groups. On the other hand statistical analysis of relative weight of liver results revealed that rats in group D1 have lowest values followed by rats in group D4. Rats in group D2 have highest values and there were no significant differences between rats in groups D3, D4, D5 and D6. Results of relative weight of kidneys showed that rats from group D1 have highest values and there was no significant difference between rats from groups D2, D3, D4, D5 and D6.
Results in Table (8) and Figure (3) showed that rats from group D5 have the lowest total cholesterol level, followed by D1, D4, D6 and D3 respectively and there was no significant difference between groups D4, D5 and D6.

Statistical analysis of serum triglycerides results showed that rats fed on diet supplemented with irradiated and non-irradiated tomato pomace have no significant difference between them (i.e., D3, D4, D5 and D6), also showed lower values than those fed on reference diet with high fat content (group D2), the same pattern observed for both VLDL-C and glucose. Results of serum LDL-C, rats in group D5 showed lowest value followed by D4, D6 and D1 respectively with no significant difference between these groups, on the other hand serum HDL-C rats fed diet supplemented with irradiated and non-irradiated tomato pomace (i.e., D3, D4, D5 and D6) have higher values with no significant difference between this groups, and serum HDL-C of rats fed on reference diet with high fat content have lowest values, atherogenic index values of rats fed on experimental diets decreased as radiation dose increase (i.e. D3 to D6), noting that there was no significant difference between those rats fed on D1, D3, D4 and rats from groups D5 and D6.

Data of liver function parameters showed that both AST and ALT of rats fed on diets supplemented with nonirradiated and irradiated tomato pomace (groups D3, D4, D5 and D6) have lower levels than rats fed on reference diet with high fat content (group D2), noting that its levels decreased by increasing radiation dose. For AST, ALT ratio there was a high significant effect of supplementing diet with irradiated and non-irradiated tomato pomace, but there was no significant difference between rats from groups D3 and D4 and rats from groups D5 and D6.

\section{Discussion:}

This study was designed to assess the effect of radiation processing on antioxidant activity of tomato pomace. Also, to assess the effect of feeding diets supplemented with these processed tomato pomace on growing rats.

Table 5: Fatty acids percentage of raw and gamma irradiated tomato pomace.

\begin{tabular}{|l|l|l|l|l|l|l|l|}
\hline \multirow{2}{*}{ Fatty acid } & \multicolumn{9}{|c|}{ Radiation Dose (kGy) } \\
\cline { 2 - 9 } & $\mathbf{0}$ & \multicolumn{2}{|c|}{$\mathbf{1 0}$} & \multicolumn{2}{|c|}{$\mathbf{2 0}$} & \multicolumn{2}{c|}{30} \\
\cline { 2 - 9 } & $\%$ & $\%$ & \% Change & $\%$ & \% Change & \% & \% Change \\
\hline Palmetic acid C16:0 & 19.26 & 17.88 & -7.17 & 18.95 & -1.61 & 21.5 & +11.63 \\
\hline Stearic acid C18:0 & 4.11 & 3.67 & -10.71 & 4.05 & -1.46 & 5.18 & +26.03 \\
\hline Oleic acid C18:1 & 27.56 & 26.61 & -3.45 & 29.72 & +7.84 & 32.56 & +18.14 \\
\hline Linoleic acid C18:2 & 46.55 & 49.95 & +7.30 & 43.82 & -5.86 & 37.19 & -20.11 \\
\hline
\end{tabular}


Table 6: Body mass of rats fed on non-irradiated and irradiated tomato Pomace incorporated in high fat diets

\begin{tabular}{|c|c|c|c|c|c|c|c|c|}
\hline \multirow{2}{*}{ Parameters } & \multicolumn{8}{|c|}{ Experimental Diets } \\
\hline & D1 & D2 & D3 & D4 & D5 & D6 & Pooled SE & P Value \\
\hline IW & $\begin{array}{l}104.71^{\mathrm{a}} \\
\pm 4.194\end{array}$ & $\begin{array}{l}111.59^{\mathrm{a}} \\
\pm 5.215\end{array}$ & $\begin{array}{r}106.2^{\mathrm{a}} \\
\pm 3.588\end{array}$ & $\begin{array}{r}98.04^{\mathrm{a}} \\
\pm 3.653\end{array}$ & $\begin{array}{l}104.34^{\mathrm{a}} \\
\pm 1.501\end{array}$ & $\begin{array}{l}111.84^{\mathrm{a}} \\
\pm 4.105\end{array}$ & 4.7013 & N.S. \\
\hline FW & $\begin{array}{l}137.79^{\mathrm{b}} \\
\pm 7.235 \\
\end{array}$ & $\begin{array}{l}229.42^{\mathrm{a}} \\
\pm 7.961\end{array}$ & $\begin{array}{l}218.04^{\mathrm{a}} \\
\pm 10.411\end{array}$ & $\begin{array}{l}222.9^{\mathrm{a}} \\
\pm 6.161\end{array}$ & $\begin{array}{c}211.9^{\mathrm{a}} \\
\pm 13.599\end{array}$ & $\begin{array}{l}205.7^{\mathrm{a}} \\
\pm 8.875\end{array}$ & 10.1655 & $<0.001$ \\
\hline TWG & $\begin{array}{r}33.08^{\mathrm{c}} \\
\pm 4.058\end{array}$ & $\begin{array}{l}118.14^{\mathrm{a}} \\
\pm 4.305\end{array}$ & $\begin{array}{l}111.84^{\mathrm{ab}} \\
\pm 7.763\end{array}$ & $\begin{array}{l}123.96^{\mathrm{a}} \\
\pm 6.774\end{array}$ & $\begin{array}{l}107.56^{\text {ab }} \\
\pm 12.190\end{array}$ & $\begin{array}{l}93.86^{\mathrm{b}} \\
\pm 5.554\end{array}$ & 7.1552 & $<0.001$ \\
\hline DWG & $\begin{array}{c}0.58^{\mathrm{c}} \\
\pm 0.075\end{array}$ & $\begin{array}{c}1.96^{\mathrm{ab}} \\
\pm 0.107\end{array}$ & $\begin{array}{c}1.93^{\mathrm{ab}} \\
\pm 0.134\end{array}$ & $\begin{array}{c}2.14^{\mathrm{a}} \\
\pm 0.118\end{array}$ & $\begin{array}{c}1.85^{\mathrm{ab}} \\
\pm 0.212\end{array}$ & $\begin{array}{c}1.62^{\mathrm{b}} \\
\pm 0.095\end{array}$ & 0.1357 & $<0.001$ \\
\hline
\end{tabular}

$\mathrm{IW}=$ Initial weight, $\mathrm{FW}=$ Final weight, $\mathrm{TWG}=$ Total weight gain, $\mathrm{DWG}=$ Daily weight gain .

Values are means \pm SE.

Means in the same Raw with the same letter are not significantly different $(\mathrm{P}<0.05)$.

Table 7: Relative weight of organs of rats fed on non-irradiated and irradiated tomato pomace incorporated in high fat diets

\begin{tabular}{|c|c|c|c|c|c|c|c|c|}
\hline \multirow{2}{*}{ Organ (\%) } & \multicolumn{8}{|c|}{ Experimental Diets } \\
\hline & D1 & D2 & D3 & D4 & D5 & D6 & Pooled SE & P Value \\
\hline Heart & $\begin{array}{c}0.35^{\mathrm{a}} \\
\pm 0.006\end{array}$ & $\begin{array}{c}0.31^{\mathrm{a}} \\
\pm 0.008\end{array}$ & $\begin{array}{c}0.31^{\mathrm{a}} \\
\pm 0.018\end{array}$ & $\begin{array}{c}0.33^{\mathrm{a}} \\
\pm 0.012\end{array}$ & $\begin{array}{c}0.31^{\mathrm{a}} \\
\pm 0.018\end{array}$ & $\begin{array}{c}0.33^{\mathrm{a}} \\
\pm 0.012\end{array}$ & 0.0131 & N.S. \\
\hline Liver & $\begin{array}{c}3.18^{\mathrm{c}} \\
\pm 0.060\end{array}$ & $\begin{array}{c}3.65^{\mathrm{a}} \\
\pm 0.111 \\
\end{array}$ & $\begin{array}{l}3.48^{\mathrm{ab}} \\
\pm 0.44\end{array}$ & $\begin{array}{c}3.34^{\mathrm{bc}} \\
\pm 0.034\end{array}$ & $\begin{array}{l}3.41^{\mathrm{abc}} \\
\pm 0.117\end{array}$ & $\begin{array}{l}3.44^{\mathrm{abc}} \\
\pm 0.034\end{array}$ & 0.0853 & $<0.01$ \\
\hline Kidney & $\begin{array}{c}0.97^{\mathrm{a}} \\
\pm 0.036 \\
\end{array}$ & $\begin{array}{c}0.80^{\mathrm{b}} \\
\pm 0.026 \\
\end{array}$ & $\begin{array}{c}0.78^{\mathrm{b}} \\
\pm 0.032 \\
\end{array}$ & $\begin{array}{c}0.77^{\mathrm{b}} \\
\pm 0.042\end{array}$ & $\begin{array}{c}0.74^{\mathrm{b}} \\
\pm 0.089 \\
\end{array}$ & $\begin{array}{c}0.78^{\mathrm{b}} \\
\pm 0.019 \\
\end{array}$ & 0.0477 & $<0.01$ \\
\hline Spleen & $\begin{array}{c}0.32^{\mathrm{a}} \\
\pm 0.015\end{array}$ & $\begin{array}{c}0.27^{\mathrm{a}} \\
\pm 0.015 \\
\end{array}$ & $\begin{array}{c}0.27^{\mathrm{a}} \\
\pm 0.003\end{array}$ & $\begin{array}{c}0.29^{\mathrm{a}} \\
\pm 0.021\end{array}$ & $\begin{array}{c}0.28^{\mathrm{a}} \\
\pm 0.018\end{array}$ & $\begin{array}{c}0.28^{\mathrm{a}} \\
\pm 0.019\end{array}$ & 0.0184 & N.S. \\
\hline Testis & $\begin{array}{c}1.40^{\mathrm{a}} \\
\pm 0.082 \\
\end{array}$ & $\begin{array}{c}1.32^{\mathrm{a}} \\
\pm 0.056 \\
\end{array}$ & $\begin{array}{c}1.23^{\mathrm{a}} \\
\pm 0.033 \\
\end{array}$ & $\begin{array}{c}1.17^{\mathrm{a}} \\
\pm 0.056 \\
\end{array}$ & $\begin{array}{c}1.17^{\mathrm{a}} \\
\pm 0.096 \\
\end{array}$ & $\begin{array}{c}1.26^{\mathrm{a}} \\
\pm 0.092 \\
\end{array}$ & 0.0845 & N.S. \\
\hline
\end{tabular}

Values are means \pm SE.

Means in the same Raw with the same letter are not significantly different.

Table 8: Serum biochemical parameters of rats fed on non-irradiated and irradiated tomato pomace incorporated in high fat diets

\begin{tabular}{|c|c|c|c|c|c|c|c|c|}
\hline \multirow{2}{*}{ Parameters } & \multicolumn{8}{|c|}{ Experimental Diets } \\
\hline & D1 & D2 & D3 & D4 & D5 & D6 & Pooled SE & $P$ Value \\
\hline $\begin{array}{l}\text { Total Cholesterol, } \\
\text { (mg/dl) }\end{array}$ & $\begin{array}{c}145.58^{\mathrm{b}} \\
\pm 1.52\end{array}$ & $\begin{array}{l}166.54^{\mathrm{a}} \\
\pm 1.802\end{array}$ & $\begin{array}{l}154.39^{\mathrm{b}} \\
\pm 0.574\end{array}$ & $\begin{array}{r}145.7^{\mathrm{c}} \\
\pm 2.490\end{array}$ & $\begin{array}{l}141.74^{\mathrm{c}} \\
\pm 1.670\end{array}$ & $\begin{array}{l}145.92^{\mathrm{c}} \\
\pm 2.562\end{array}$ & 1.891 & $<0.001$ \\
\hline $\begin{array}{l}\text { Triglycerides, } \\
\text { (mg/dl) }\end{array}$ & $\begin{array}{l}85.03^{\mathrm{c}} \\
\pm 0.815\end{array}$ & $\begin{array}{l}120.87^{\mathrm{a}} \\
\pm 4.666\end{array}$ & $\begin{array}{l}105.31^{\mathrm{b}} \\
\pm 2.674\end{array}$ & $\begin{array}{l}104.46^{\mathrm{b}} \\
\pm 0.579\end{array}$ & $\begin{array}{l}100.9^{\mathrm{b}} \\
\pm 3.620\end{array}$ & $\begin{array}{l}100.61^{\mathrm{b}} \\
\pm 0.262\end{array}$ & 2.288 & $<0.001$ \\
\hline HDL-C, (mg/dl) & $\begin{array}{c}99.7^{\mathrm{b}} \\
\pm 0.268\end{array}$ & $\begin{array}{l}95.58^{\mathrm{c}} \\
\pm 0.054\end{array}$ & $\begin{array}{l}101.65^{\mathrm{a}} \\
\pm 0.883\end{array}$ & $\begin{array}{l}102.26^{\mathrm{a}} \\
\pm 0.793\end{array}$ & $\begin{array}{l}102.37^{\mathrm{a}} \\
\pm 0.671\end{array}$ & $\begin{array}{l}102.68^{\mathrm{a}} \\
\pm 0.305\end{array}$ & 0.650 & $<0.001$ \\
\hline LDL -C, (mg/dl) & $\begin{array}{l}26.2^{\mathrm{bc}} \\
\pm 0.406\end{array}$ & $\begin{array}{l}47.14^{\mathrm{a}} \\
\pm 3.729\end{array}$ & $\begin{array}{l}31.73^{b} \\
\pm 0.614\end{array}$ & $\begin{array}{l}22.55^{\mathrm{c}} \\
\pm 1.942\end{array}$ & $\begin{array}{l}21.96^{\mathrm{c}} \\
\pm 1.148\end{array}$ & $\begin{array}{l}23.11^{\mathrm{c}} \\
\pm 2.688\end{array}$ & 2.451 & $<0.001$ \\
\hline VLDL-C，(mg/dl) & $\begin{array}{c}17.0^{\mathrm{d}} \\
\pm 0.162\end{array}$ & $\begin{array}{c}24.2^{\mathrm{a}} \\
\pm 0.939\end{array}$ & $\begin{array}{l}21.05^{\mathrm{b}} \\
\pm 0.537\end{array}$ & $\begin{array}{c}20.9^{\mathrm{b}} \\
\pm 0.119\end{array}$ & $\begin{array}{l}20.18^{\mathrm{b}} \\
\pm 0.718\end{array}$ & $\begin{array}{l}20.12^{\mathrm{b}} \\
\pm 0.057\end{array}$ & 0.457 & $<0.001$ \\
\hline Atherogenic Index & $\begin{array}{c}0.43^{\mathrm{b}} \\
\pm 0.004\end{array}$ & $\begin{array}{c}0.75^{\mathrm{a}} \\
\pm 0.022\end{array}$ & $\begin{array}{c}0.5^{\mathrm{b}} \\
\pm 0.00\end{array}$ & $\begin{array}{c}0.46^{\mathrm{b}} \\
\pm 0.018\end{array}$ & $\begin{array}{c}0.4^{\mathrm{c}} \\
\pm 0.00\end{array}$ & $\begin{array}{c}0.37^{\mathrm{c}} \\
\pm 0.021\end{array}$ & 0.016 & $<0.001$ \\
\hline Glucose, (mg/dl) & $\begin{array}{l}90.01^{\mathrm{d}} \\
\pm 2.178\end{array}$ & $\begin{array}{c}122.73^{\mathrm{a}} \\
\pm 0.20\end{array}$ & $\begin{array}{l}118.21^{b} \\
\pm 0.267\end{array}$ & $\begin{array}{l}115.8^{b} \\
\pm 0.30\end{array}$ & $\begin{array}{l}116.53^{b} \\
\pm 0.219\end{array}$ & $\begin{array}{l}116.53^{b} \\
\pm 0.128\end{array}$ & 0.952 & $<0.001$ \\
\hline AST, (U/L) & $\begin{array}{l}37.92^{\mathrm{c}} \\
\pm 0.115\end{array}$ & $\begin{array}{l}42.63^{\mathrm{a}} \\
\pm 0.036\end{array}$ & $\begin{array}{l}40.50^{\mathrm{c}} \\
\pm 0.134\end{array}$ & $\begin{array}{l}40.88^{\mathrm{c}} \\
\pm 0.222\end{array}$ & $\begin{array}{l}40.83^{\mathrm{c}} \\
\pm 0.295\end{array}$ & $\begin{array}{l}40.83^{\mathrm{c}} \\
\pm 0.314\end{array}$ & 0.163 & $<0.001$ \\
\hline ALT, (U/L) & $\begin{array}{l}38.18^{\mathrm{e}} \\
\pm 0.13\end{array}$ & $\begin{array}{l}44.68^{\mathrm{a}} \\
\pm 0.125\end{array}$ & $\begin{array}{l}43.79^{b} \\
\pm 0.006\end{array}$ & $\begin{array}{l}41.33^{\mathrm{c}} \\
\pm 0.199\end{array}$ & $\begin{array}{l}37.27^{1} \\
\pm 0.272\end{array}$ & $\begin{array}{l}39.33^{\mathrm{d}} \\
\pm 0.095\end{array}$ & 0.163 & $<0.001$ \\
\hline AST, ALT ratio & $\begin{array}{c}0.99^{\mathrm{b}} \\
\pm 0.003\end{array}$ & $\begin{array}{c}0.95^{\mathrm{c}} \\
\pm 0.004\end{array}$ & $\begin{array}{c}0.89^{\mathrm{d}} \\
\pm 0.009\end{array}$ & $\begin{array}{c}0.88^{\mathrm{d}} \\
\pm 0.009\end{array}$ & $\begin{array}{c}1.08^{\mathrm{a}} \\
\pm 0.022\end{array}$ & $\begin{array}{c}1.07^{\mathrm{a}} \\
\pm 0.004\end{array}$ & 0.008 & $<0.001$ \\
\hline
\end{tabular}

Values are means \pm SE.

Means in the same Raw with the same letter are not significantly different $(\mathrm{P}<0.05)$. 


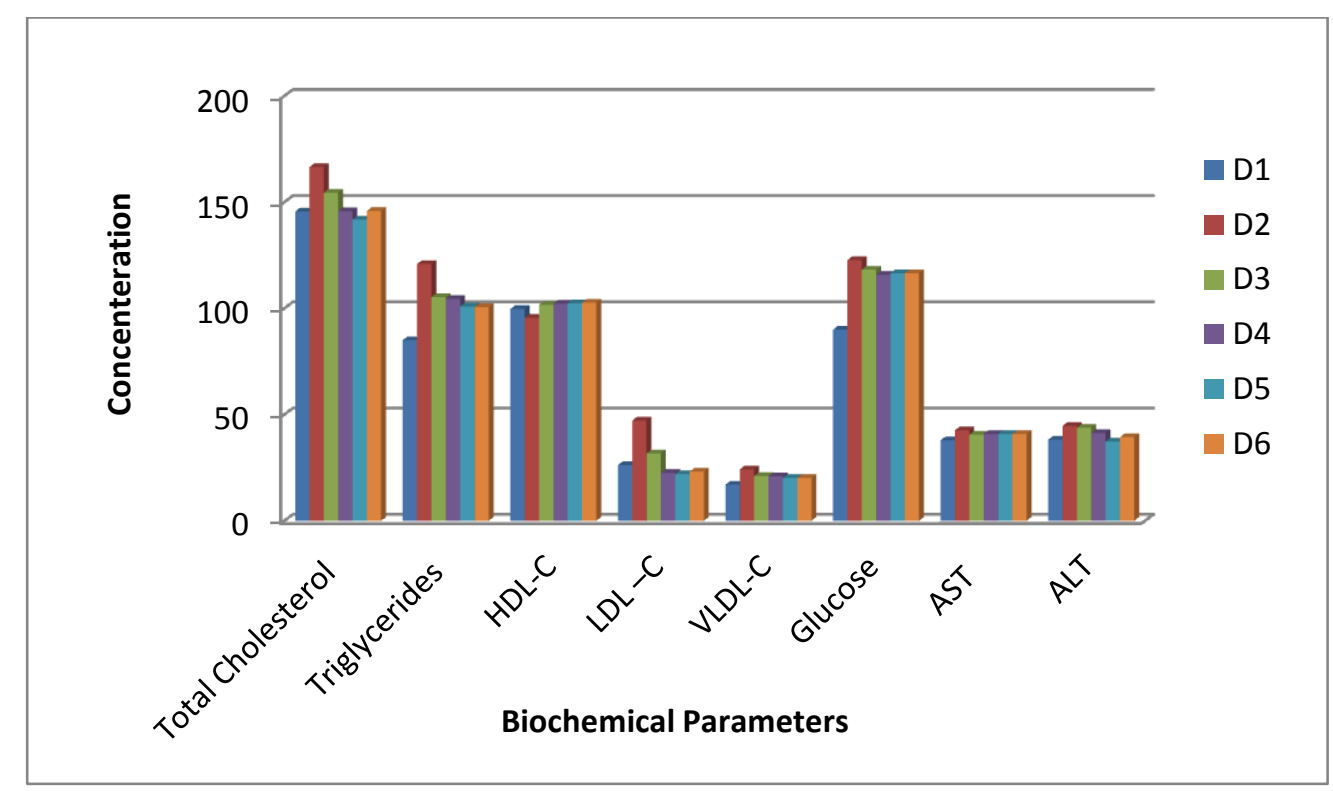

Figure (3): Biochemical parameters of rats fed on non-irradiated and irradiated tomato pomace incorporated in high fat diets

Results of the effect of irradiation on chemical composition of tomato pomace revealed that there were no significant differences in most nutrients in the chemical composition between non-irradiated and irradiated samples at dose levels of 10, 20 and $30 \mathrm{kGy}$, this can be attributed to the relatively limited amount of water content (about 13.6\%), so it would not be easy to be radiolyzed by irradiation to produce enough free radicals that could induce significant changes in gross composition of these material. Moreover, the crude protein and fat in a complex matrix of foodstuffs have been reported to be more resistant to radiation than in the pure state ${ }^{[34]}$. There was a significant changes in ash content from statistical analysis point of view, however from practical point of view these rear changes was not significant, crud fiber of tomato pomace was significantly decreased as radiation dose increase from $10 \mathrm{kGy}$ to $30 \mathrm{kGy}$. On the other hand, nitrogen free extract (NFE) content increases as radiation dose increase, these can be attributed to break down of fiber molecules as the absorbed energy increases leading to the formation of compounds of lower molecular weight, this explanation agrees with Hussain, et al., ${ }^{[35]}$ who irradiated four bean varieties (red, yellow, black and white), by $\gamma$ - rays at dose levels 5 to $25 \mathrm{kGy}$.

Results agrees with Ashraf ${ }^{[36]}$ who studied the effect of processing soybean, broad bean and pea by ionizing irradiations (gamma and electron beam rays) at dose levels 5, 10, 30 and $60 \mathrm{kGy}$ of both ray sources, and in good agreement with previous studies by El-Niely ${ }^{[37]}$, Ali ${ }^{[38]}$ and Farag ${ }^{[39]}$. In that irradiation processing didn't cause significant changes in the chemical composition of soybean and broad bean agro industrial wastes at the applied radiation doses.

In the present study the total phenols of processed tomato pomace was increased and the most pronounced effect was noticed for tomato pomace irradiated by 10 $\mathrm{kGy}$ of $\gamma$-rays as compared to non-irradiated samples, on the other hand tannins in tomato pomace was decreased in sample processed by 20 and $30 \mathrm{kGy}$.

Phenolic compounds can range in size from monomers to long-chain polymers such as tannins, and usually exist bound to carbohydrates or as part of repeating subunits of high molecular weight polymers ${ }^{[40]}$, so the decrease or increase in tannins content can be explained by that, irradiation processing may cause polymerization of small molecular weight compound leading to the formation of higher molecular weight or breakdown of large molecular weight leading to the formation of low molecular weight polyphenols as occurred in processed tomato pomace samples.

Results in the present study of polyphenols agree with Harrison and Were ${ }^{[40]}$ who mentioned that $\gamma$ irradiation increased the total phenolics yield in almond skin extracts $45 \%$ at dose levels of $4 \mathrm{kGy}$ and above when compared to the control (non-irradiated), and explained their results to the release of phenolic compounds from glycosidic components and the degradation of larger phenolic compounds into smaller ones by $\gamma$ - irradiation.

Also, the current results agree with previous studies which reported the decrease in tannins level in different types of legumes using $\gamma$ - irradiation, ${ }^{[41,42]}$. Behgar, $\boldsymbol{e t}$ al. ${ }^{[43]}$ found that $\gamma$-irradiation with doses between 10-60 $\mathrm{kGy}$ caused the decreased tannin content of the pistachio hull extracts, he also, found that samples irradiated by doses between $20 \mathrm{kGy}$ and $60 \mathrm{kGy}$ the level of total phenolics increased compared to the control, the same author put an explanation of the variable effects of ionizing radiation on polyphenols and tannins; that irradiation processing may cause higher extractability of these compounds in irradiated samples as a result of 
alternations in cellular compounds and release of bound or insoluble phenolics especially at high doses of irradiation.

In most studies using ionizing irradiation treatment of food stuffs, a decrease in the level of tannin was accompanied with an increase in total phenolic compounds ${ }^{[40,44,45]}$.

DPPH radical is widely used to test the radical scavenging ability and therefore the antioxidant activity in both simple compounds and plant extracts. The irradiation processing of tomato pomace caused a decrease in DPPH scavenging activity, the most pronounced effect was by $10 \mathrm{kGy}$ compared to nonirradiated samples. The effect on tomato pomace can be explained by the breakdown of ascorbic acid, which is the most sensitive vitamin to irradiation processing ${ }^{[46]}$.

The present results are in line with previous reports, Pérez, et al. ${ }^{[47]}$, who observed that scavenging activity of control pistachio hull higher by $(53.96 \%)$ than those of irradiated samples (10-60 kGy). $\gamma$-irradiation (5-30 $\mathrm{kGy}$ ) significantly decreased the DPPH radicalscavenging activity of ground black pepper extracts ${ }^{[48]}$. On the other hand, Kim et al. ${ }^{[45]}$, reported that ionizing irradiation of citrus pomaces increased scavenging activity of citrus pomaces extracts in methanol, ethanol and water at the absorbed doses of 3.6-37.9 kGy, and on increasing the dose of irradiation, scavenging activity in acetone and water extract of citrus pomace was decreased.

Plant and food materials are very complex, the overall changes observed in irradiated samples can be summarized in that, radiation processing may cause tannins breakdown leading to decrease antioxidant activity or these reduction lead to increase smaller weight polyphenols, which leads to increase antioxidant activity. Another explanation is that, irradiation processing leads to breakdown of radiosensitive compounds as vitamin $\mathrm{C}$ with antioxidative activity which leads to decrease in antioxidant activity.

The quality of protein is known to be affected by essential amino acid composition, amino acid imbalance, biological availability of essential amino acids and digestibility. In current study, there were significant increase in TAA, TEAA, EAAI and BV levels, while a significant decrease in TNEAA levels. In tomato pomace processed by $30 \mathrm{kGy}$ TNEAA was increased.

These results agree with previous results obtained by Ashraf, [36] who studied the effect of processing soybean, broad bean and pea by ionizing irradiations (gamma and electron beam rays) at dose level $60 \mathrm{kGy}$ of both rays; Farag and EL-Niely ${ }^{[49]}$, who used $\gamma$ - rays at dose level $25 \mathrm{kGy}$ on dried tomato pomace; ${ }^{[50]}$ on amino acids of baby food irradiated by $\gamma$ - rays at dose levels of $0.5,1.5,6,10,30,50 \mathrm{kGy}$, and Kausar et al. $^{[51]}$ who studied the effects of $\gamma$ - irradiation at dose level 5 and $10 \mathrm{kGy}$ on amino acids present in Camellia sinensis, in that the amino acid content were decreased for some amino acids and increased for another with no fixed pattern.

Also, Lotto et al. ${ }^{[52]}$, observed a fluctuating changes either by loss or increase in the amino acid values of beans, pea and lupine seeds, the magnitude of the changes was correlated with the dose level, the general effect of $\gamma$-irradiation processing on amino acid analysis can be summarized in that, sulfur-containing and aromatic acids are the most sensitive to irradiation, while simple amino acids could be formed by the destruction of complicated amino acids ${ }^{[53]}$, increased or decreased content of amino acids with increasing irradiation dose with no clear trend of the change, these effects could be related to the structure of amino acids. Where, simple amino acids due to irradiation undergo reductive deamination and decarboxylation ${ }^{[54]}$.

Changes in the concentration of amino acids induced by irradiation may probably be due to free radicals that might be formed in association with splitting of the peptide bonds, deamination and decarboxylation reactions of amino acids followed by chains of chemical reactions forming other new radicals ${ }^{[55]}$.

Results of fatty acids analysis showed that irradiation processing at all doses used caused significant effects on fatty acids profile of tomato pomace, these effects appears in increase and decrease of fatty acids with no fixed pattern, but the overall effect in most processed samples was the increase in essential fatty acids. The fluctuating values of fatty acids in samples processed by $\gamma$ - irradiation may be because irradiation affects the yield of extracted oil, these results agree with Silva et al., ${ }^{[57]}$, Afify et al. ${ }^{[58]}$, El-Niely ${ }^{[59]}$ and Olotu, et al. ${ }^{\left[{ }^{60]},\right.}$, who used $\gamma$-radiation, where irradiation caused the formation of free radicals which react with poly unsaturated fatty acids producing trans fatty acids, unstable hydroperoxides and a range of further degradation products.

The determination of body and organ relative weight was used as a sensitive index of toxicity that may have resulted from the consumption of any food material. Organ weight is also considered to be a good indicator of the general health status of animals fed with feed ingredients treated with ionizing radiation ${ }^{[61]}$.

Rats fed on reference diet for 8 weeks had the lowest final weight, total weight gain and daily weight gain. On the other hand, rats fed on reference diet with $20 \%$ fat and diets supplemented with non-irradiated and irradiated tomato pomace at all dose levels used, for 8 weeks have higher values $>3$ folds for the three parameters, one of reasons is the high fat content (> 20\% fats), and there were no significant differences between rats fed on non-irradiated and irradiated tomato pomace diets in these parameters.

The most pronounced effect of feeding irradiated samples was for rats fed on diet supplemented with tomato pomace irradiated by $10 \mathrm{kGy}$ of $\gamma$-rays, followed by rats fed on diets supplemented by tomato pomace irradiated by 0,20 and $30 \mathrm{kGy}$, this may be attributed to that tomato pomace contains the lowest content of tannins which is considered as an antinutritional factor 
beside its activity as strong antioxidant, these agrees with Tebib et al. ${ }^{[62]}$, who explained the mode of action of tannins, is by aggregation and precipitation of proteins inducing significant amounts of undigested material released in feces ${ }^{[63-65]}$.

Results of relative weight of liver, showed a significant difference between rats fed on experimental diets, where, rats fed on diets supplemented with nonirradiated and irradiated tomato pomace and $20 \%$ fat, showed values between values obtained from rats fed on reference diets and rats fed on reference diet with $20 \%$ fat, these may be due to the stress caused by the presence of high fat content $>20 \%$ in diets of groups fed on diets supplemented with non-irradiated and irradiated tomato pomace and $20 \%$ fat, but these stress was highly neutralized by the presence of both high fiber content and bioactive compounds (antioxidants) in tomato pomace, this explanation agrees with Walugembe et al. ${ }^{[66]}$, El-Neily and El-Shenawy ${ }^{[67]}$, Pereira et al. ${ }^{[68]}$ and Sho et al. ${ }^{[69]}$.

In the present study rats fed on reference diet with $20 \%$ fat had the highest values of tested parameters as serum cholesterol, triglycerides, low density lipoprotein cholesterol (LDL-C), very low lipoprotein cholesterol (VLDL-C), atherogenic index (AI), glucose, liver function parameters (AST, ALT) and AST/ALT ratio, while high density lipoprotein cholesterol (HDL-C) showed lowest values, leading to higher risk for atherosclerosis and coronary heart disease. On the other hand, supplementing high fat diets with non-irradiated or irradiated tomato pomace had positive effects on the health of rats fed on it.

Results are in good agreement with Jiao et al., ${ }^{[70]}$, who observed that feeding hamsters with high fat diet supplemented with tomato by-products (tomato pomace, defatted tomato pomace and tomato seed oil) caused a significant decrease in serum LDL-C, TG and increase in HDL-C. Hsu et al. ${ }^{[71]}$, also reported that consumption of $3 \%$ and $9 \%$ tomato paste significantly increased serum HDL-C level after 8 weeks of feeding high fat diet.

The present results are in line with those obtained by Kunyanga, et al., ${ }^{[72]}$, who revealed that tannins in amaranth grain, finger millet, field bean, sunflower seeds, drumstick, and amaranth leaves specially condensed ones as present in tomato pomace has a strong antioxidant and antidiabetic activities.

The hypoglycemic effect of supplementing high fat diets with non-irradiated or irradiated tomato pomace agrees with El-Alfy, et al. ${ }^{[73]}$ who found that, grape seeds extract caused significant decline in the hyperglycemia also, caused increase in serum insulin levels in diabetic rats, and explained this effect because their potent antioxidant properties. The present study demonstrated that tomato pomace contains compounds with potent antioxidant activities, which can exert antidiabetic effects by preserving pancreatic cell function. The results of serum liver functions of rats fed on diet supplemented with non-irradiated and irradiated tomato pomace agrees with studies performed by Ibrahim et al., ${ }^{[74]}$, Hsu et al. ${ }^{[75]}$ and Kim, et al. ${ }^{[76]}$, who reported that supplementation of high fat diet with tomato pomace extract significantly reduced serum AST and ALT of rats.

Conclusion: The results from both physiological and biochemical responses of growing male Wister rats kept for 8 weeks on diets supplemented with non-irradiated or irradiated tomato pomace showed that there were no critical changes observed due to irradiation. Further research needs to be conducted to estimate the individual effect of radiation processing on each bioactive compound present in the tomato pomace extract.

\section{References}

1) Cho, H. Y. and Kleeberger, S. R. (2007). Genetic mechanisms of susceptibility to oxidative lung injury in mice. Free Radical Biology and Medicine, 42(4): 433-445.

2) Migliore, L. and Coppedè, F. (2009). Environmental-induced oxidative stress in neurodegenerative disorders and aging. Mutation Research, 674: 73-84.

3) Durackova, Z. (2010). Some current insights into oxidative stress. Phys. Res. 59: 459-469.

4) Sasipriya, G. and Siddhuraju, P. (2012). Effect of different processing methods on antioxidant activity of underutilized legumes, Entada scandens seed kernel and Canavalia gladiata seeds. Food Chem Toxicol 50(8): 2864-2872.

5) Reuter, S., Gupta, S. C., Chaturvedi, M. M. and Aggarwal, B. B. (2010). Oxidative stress, inflammation, and cancer: how are they linked? Free Radic. Biol. Med. 49: 1603-1616.

6) Schieber, A., Stintzing, F. C. and Carle, R. (2001). By-products of plant food processing as a source of functional compounds - recent developments. Trends in Food Science and Technology, 12: 401413.

7) Moure, A., Cruz, J. M., Franco, D., Domínguez, J.M., Sineiro, J. and Dominguez, H. (2001). Natural antioxidants from residual sources. Food Chemistry, 72: 145-171.

8) Peschel, W., Sánchez-Rabaneda, F., Diekmann, W., Plescher, A., Gartzía, I. and Jiménez, D. (2006). An industrial approach in the search of natural antioxidants from vegetable and fruit wastes. Food Chemistry, 97: 137-150.

9) Ramarathnam, N., Osawa, T., Namiki, M. and Kawakishi, S. (1989). Chemical studies on novel rice hull antioxidants. 2. Identification of isovitexin, a C-glycosyl flavonoid. Journal of Agricultural and Food chemistry, 37: 316-319.

10) Watanabe, M., Ohshita, Y. and Tsushida, T. (1997). Antioxidant compounds from buckwheat (Fagopyrum esculentum) hulls. Journal of Agricultural and Food Chemistry, 45: 1039-1044. 
11) Takeoka, G. R. and Dao, L. T. (2002). Antioxidant constituents of almond [Prunus dulcis (Mill.) D.A. Webb] hulls. Journal of Agricultural and Food Chemistry, 51: 496-501.

12) Kabir, F., Tow, W. W., Hamauzu, Y., Katayama, S., Tanaka S. and Nakamura, S. (2015). Antioxidant and cytoprotective activities of extracts prepared from fruit and vegetable wastes and byproducts. Food Chem 167: 358-362.

13) Navarro-González, I., García-Valverde, V., García-Alonso, J. and Periago, M. J. (2011). Chemical profile, functional and antioxidant properties of tomato peel fiber. Food Research International 44(5): 1528-1535.

14) Rizk, E. M., El-Kady, A. T. and El-Bialy, A. R. (2014). Charactrization of carotenoids (lyco-red) extracted from tomato peels and its uses as natural colorants and antioxidants of ice cream. Annals of Agricultural Sciences 59(1): 53-61.

15) Khattak, K. F., Simpson, T. J. and Ihasnullah (2009). Effect of gamma irradiation on the microbial load, nutrient composition and free radical scavenging activity of Nelumbonucifera rhizome. Radiation Physics and Chemistry, 78: 206-212.

16) Dacie, J. V. and Lewis, S. M. (1975). Practical Haematology. Churchill, Livingstone, ED. binburgh, London and NY, $5^{\text {th }}$ ed.

17) A.O. A. C. (1995). Association of Official Analytical Chemists International $16^{\text {th }}$ ed., Arlington, Virginia, USA.

18) A.O. A. C. (2000). Association of Official Analytical Chemists International $17^{\text {th }}$ ed., Arlington, Virginia, USA.

19) Hamilton, R. J. and Hamilton, S. (1993). Lipid analysis a practical approch. IRL press, Oxford University Press, Oxford.

20) Suzanna and Nielson, S. (1998). Food analysis, second edition, An Aspen puplication, Aspen publishers, inc., Gaithersburg, Mary Lan. P. 34.

21) Nellet Leo, M. L. (1996). Hand book of food analysis, Vol.1 physical characterization and nutrient analysis, chapter (7): p. 210, Marcel Dekkes, Inc.

22) Balbaa, S. I. (1974). Chemistry of crude drugs. Laboratory manual, Al-Shaab Printing House, Cairo, Egypt, p. 1-194.

23) Blois, M. S. (2002). Antioxidant determinations by the use of a stable free radical. Nature 26: 11991200.

24) Daniel, H. D. and George, C. M. (1972). Peach seed dormancy in relation to indigenous inhibitors and applied growth substances. J. Amer. Soc. Hort. Sci. 97: 651-654.

25) Sherwin, J. E. Liver function. In: Kaplan, L. A. and Pesce, A. J. (1984). eds. Clinical chemistry, theory, and correlation. St. Louis: Mosby; 420-438.

26) Richmond, N., (1973). Preparation and properties of a cholesterol oxidase from Nocardia sp. and its application to the enzymatic assay of total cholesterol in serum. Clin. Chem.; 19:1350-1356.

27) Lopes-virella, M. F. (1977). Cholesterol determina- tion in high-density lipoproteins separated by three different methods. Clin. Chem.; 23: 882.

28) Mgowan, M. W., Artiss, J. D., Standbergh, D. R. and Zak, B. A. (1983). Peroxidase-coupled method for colorimetric determination of serum triglycerides. Clin. Chem.; 29:538-452.

29) Ahmedi, S. A., Boroumand, M. A., Moghddam, K. G., Tajik, P. and Dibaj, S. M., (2008). The impact of low-serum triglyceride on LDL-cholesterol estimation. Arch. Iran Med., 11: 318-321.

30) Satheesh, M. and Pari, L., (2008). Effect of pterostibene on lipid and lipid profile in streptozotocin-nicotinamide type 2 diabetes mellitus. J. Appl. Biomed., 6: 31-37.

31) Pandya, N., Santani, D. and Jain, S., (2006). Antioxidant activity of ezetimibe in hyper cholesterolemic rats. Indian J., 38: 205-206.

32) Caraway, W. T. and Watts, N. B. (1987). Carbohydrates In: Tietz NW, ed. Fundamentals of Clinical Chemistry. $3^{\text {ry }}$ ed. Philadelphia WB Saunders: in group 422-447.

33) Duncan, D. B. (1955). Multiple range tests and multiple F-test. Biometrica, 11:1.

34) Diehl, J. F. and Scherz, H. (1975). Estimation of radiolytic products as a basis for evaluation the wholesomeness of irradiated foods. Int. J. Appl. Rad. Isotopes, 26:499.

35) Hussain, P. R., Wani, I. A., Suradkar, P. P. and Dar, M. A. (2014). Gamma irradiation induced modification of bean polysaccharides: impact on physicochemical, morphological and antioxidant properties. Carbohydrates Polymers, 110: 183-194.

36) Mounir, A. M. (2008). The possible biochemical effects of radiation processing in certain food enriched with reducing sugars. M.Sc. Thesis, Dept. Biochemistry, Faculty of Science. Ain- Shams Univ.

37) El-Niely, H. F. (1996). Chemical constituent and digestibility of broad beans subjected to irradiation treatment. M.Sc. Thesis. Biochemistry and Nutrition Department, Women's College, Ain Shams Univ. Egypt.

38) Ali, S. Y. (1997). Effect of irradiation some plant feed stuffs on some nutritive and physiological parameters in chickens. M.Sc. Thesis, Animal Production Department, Fac. of Agri., AL Azhar Univ., Cairo, Egypt.

39) Farag, M. D. H. (1998). The nutritive value for chicks of full-fat soybeans irradiated at up to $60 \mathrm{kGy}$. J. Animal Feed Sci. Tech. 73:319.

40) Harrison, K. and Were, L. (2007). Effect of gamma irradiation on total phenolic content yield and antioxidant capacity of Almond skin extracts. Food Chemistry 102(3): 932-937.

41) El-Niely, H. F. G. (2007). Effect of radiation processing on antinutrients, in vitro protein digestibility and protein efficiency ratio bioassay of legume seeds. Radiat. Phys. Chem. 76:1050-1057.

42) De Toledo, T. C. F., Canniatti-Brazaca, S. G., Arthur, V. and Piedade, S. M. S. (2007). Effects of $\gamma$-radiation on total phenolics, trypsin and tannin 
inhibitors in soybean grains. Radiat. Phys. Chem. 76: 1653-1656.

43) Behgar, M., Ghasemib, S., Naserian, A., Borzoie, A. and Fatollahi, H. (2011). $\gamma$-radiation effects on phenolics, antioxidants activity and in vitro digestion of pistachio (Pistachia vera) hull. Journal of Radiation Physics and Chemistry, 80: 963-967.

44) Stajner, D., Milosevic, M. and Popovic, B. M., (2007). Irradiation effects on phenolic content, lipid and protein oxidation and scavenger ability of soybean seeds. Int. J. Mol. Sci., 8: 618-627.

45) Kim, J., Lee, B. C., Lee, J., Nam, K. and Lee, S. (2008). Effect of electron-beam irradiation on the antioxidant activity of extracts from Citrus unshiu pomaces. Radiat. Phys. Chem., 77: 87-91.

46) Kilcast, D. (1994). Effect of irradiation on vitamins. Food Chem., 49: 157-164.

47) Pérez M. B., Banek S. A. and Croci, C. A. (2011). Retention of antioxidant activity in $\gamma$-irradiated Argentinian sage and oregano. Journal of Food Chemistry, 126: 121-126.

48) Suhaj, M., Rácová, J., Polovka, M. and Brezová, V. (2006). Effect of $\gamma$-irradiation on antioxidant activity of black pepper (Piper nigrum L.). Food Chemistry, 97(4): 696-704.

49) Farag, M. D. H. and El-Niely, H. F. (2006) Bioconversion of radiation processed dried tomato pomace to high protein animal feed. Egypt. J. Rad. Sci. Applic., 19(1).

50) Matloubi, H., Aflaki, F. and Hadjiezadegan, M. (2004). Effect of $\gamma$-irradiation on amino acids content of baby food proteins. Journal of Food Composition and Analysis, 17(2): 133-139.

51) Kausar, T., Akram, K. and Kwon, J. H. (2013). Comparative effects of irradiation, fumigation, and storage on the free amino acids and sugar contents of green, black and oolong teas. Radiation Physics and Chemistry, 86: 96-101.

52) Lotto, G., Anelli, G., Lomora, A. and Teglia, A. (1974). Effect of $\gamma$ - irradiation on leguminous seed protein. Sci. Tech. Aliment.

53) Elias, P. S. and Cohen, A. J. (1997). Radiation Chemistry of Major Food Components. Elsevier Scientific, Amsterdam.

54) Urbian, W. M. (1986). Food Irradiation. Academic Press, Orlando, FL.

55) Liebster, J. and Kapoldova, J. (1964). The radiation chemistry of amino acids. Advances in Radiation Biology, 1: 157.

56) Elias, P. S. and Cohen, A. J. (1977). Radiation chemistry of major food components. Netherlands: Elsevier Biomedical.

57) Silva, T. M., Miranda, R. R. S., Ferraz, V. P., Pereira, M. T., de Siqueira, E. P. and Alcântara, A. F. C. (2013). Changes in the essential oil composition of leaves of Echinodorus macrophyllus exposed to $\gamma$-radiation. Revista Brasileira de Farmacognosia, 23(4): 600-607.

58) Afify, A. M. R., Rashed, M. M., Ebtesam, A. M. and El-Beltagi, H. S. (2013). Effect of $\gamma$-radiation on the lipid profiles of soybean, peanut and sesame seed oils. Fats Oils, 64:356-368.

59) El-Niely, H. F. G. (2001). Biochemical and nutritional studies on radiation processed peanuts, Ph.D. Thesis, Biochemistry and Nutrition Department, Women's College, Ain Shams University, Cairo, Egypt.

60) Olotu, I., Enujiugha, V., Obadina, A. and Owolabi, K. (2014). Fatty acid profile of $\gamma$-irradiated and cooked African oil bean seed (Pentaclethra macrophylla Benth). Food Sci. Nutr., 2(6): 786-791.

61) Farag, M. D. H. (1987). Studies on protein turnover in poultry fed irradiated protein by-products. Ph.D. Thesis, Faculty of Agriculture, Al-Azhar University, Cairo, Egypt.

62) Katia, T., Pierre, B. and Jean-Max, R. (1996). Effects of dietary grape seed tannins on rat cecal fermentation and colonic bacterial enzymes. Journal of Nutrition Research, 16(1): 105-110.

63) Vallet, J., Rouanet, J. M. and Besancon, P. (1994). Dietary grape seed tannins :effects on nutritional balance and on some enzymic activities along the crypt-villus axis of rat small intestine. Ann Nutr. Metab, 38: 75-84.

64) Makker, H. P. S., Singh, B. and Dawra, R. K. (1987). Tannin-nutrients interaction - A review. Int. J. Anim. Sci., 2: 127-140.

65) Hagerman, A. E. and Butler, J. C. (1980). Determination of protein in tannin-protein precipitate. J. Agr. Food Chem, 28: 944-947.

66) Walugembe, M., Rothschild, M. F. and Persia, M. E. (2014). Effects of high fiber ingredients on the performance, metabolizable energy and fiber digestibility of broiler and layer chicks. Animal Feed Science and Technology, 188: 46-52.

67) El- Neily, H. F. G. and El-Shenawy, H. M. (2011). Effect of grape seeds oil extracted from radiation processed seeds on lipid metabolism and on antioxidant activity in rats fed on diets containing cholesterol. J. of isotope \& rad. Res., 43(3): 801-823.

68) Pereira, C., Calhelha, R. C., Barros, L. and Ferreira, I. C. F. R. (2013). Antioxidant properties, anti-hepatocellular carcinoma activity and hepatotoxicity of artichoke, milk thistle and borututu. Industrial Crops and Products 49: 61-65.

69) Sho, D., Bartey Glenn, E., Yokoyama, W., Pan, Z., Zhang, H. and Zhang, A., (2013). Plasma and hepatic cholesterol-lowering effects of tomato pomace, tomato seed oil and defatted tomato seed in hamsters fed with high fat diets, J. Food Chemistry, 139: $589-596$.

70) Jiao, R., Zhang, Z., Yu, H., Huang, Y. and Chen Z. Y. (2010). Hypocholesterolemic activity of grape seed proanthocyanidin is mediated by enhancement of bile acid excretion and up-regulation of CYP7A1. J. of Nutritional Biochemistry, 21: 1134-1139.

71) Hsu, Y., Lai, C., Chang, C., Fan, C., Chen, C. and Wu, C. (2008). Characterizing the lipid lowering effects and antioxidant mechanisms of tomato paste. Bioscience, Biotechnology and Biochemistry, 72(3): 677-685. 
72) Kunyanga, C. N., Imungi, J. K., Okoth, M., Momanyi, C., Biesalski, H. K. and Vadivel, V. (2011). Antioxidant and antidiabetic properties of condensed tannins in acetonic extract of selected raw and processed indigenous food ingredients from Kenya. J. Food Sci., 76(4): 560-567.

73) El-Alfy, A. T., Ahmed, A. A. and Fatani, A. J. (2005). Protective effect of red grape seeds proanthocyanidins against induction of diabetes by alloxan in rats. Pharmacol Res., 52(3): 264-270.

74) Ibrahim, H. S., Ahmed, L. A. and El-din, M. M. E. (2008). The functional role of some tomato products on lipid profile and liver function in adult rats. Journal of Medicinal Food, 11(3): 551-559.

75) Hsu, Y., Lai, C., Chang, C., Fan, C., Chen, C. and Wu, C. (2008). Characterizing the lipid lowering effects and antioxidant mechanisms of tomato paste. Bioscience, Biotechnology and Biochemistry, 72(3): 677-685.

76) Kim, A. Y., Jeong, Y. J. , Park, Y. B., Lee, M.-K., Jeon, S. M., McGregor, R. A. and Choi, M. S. (2012). Dose dependent effects of lycopene enriched tomato-wine on liver and adipose tissue in high-fat diet fed rats. Food Chemistry, 130(1): 42-48. 